\section{DUAL BLOCKADE OF THE PD-1 CHECKPOINT PATHWAY AND THE ADENOSINERGIC NEGATIVE FEEDBACK SIGNALING PATHWAY WITH A PD-1/CD73 BISPECIFIC ANTIBODY FOR CANCER IMMUNE THERAPY}

Tingting Zhong, Zhaoliang Huang, Xinghua Pang, Na Chen*, Xiaoping Jin, Yu Xia, Zhongmin Maxwell Wang, Baiyong Li, Yu Xia. Akeso Biopharma Co., Ltd., Zhongshan city, China

Background CD73 (ecto-5'-nucleotidase) is an ecto-nucleotidase that dephosphorylate AMP to form adenosine. Activation of adenosine signaling pathway in immune cells leads to the suppression of effector functions, down-regulate macrophage phagocytosis, inhibit pro-inflammatory cytokine release, as well as yield aberrantly differentiated dendritic cells producing protumorigenic molecules. ${ }^{1}$ In the tumor microenvironment, adenosinergic negative feedback signaling facilitated immune suppression is considered an important mechanism for immune evasion of cancer cells. ${ }^{2}{ }^{3}$ Combination of CD73 and anti-PD1 antibody has shown promising activity in suppressing tumor growth. Hence, we developed AK119, an anti- human CD73 monoclonal antibody, and AK123, a bi-specific antibody targeting both PD-1 and CD73 for immune therapy of cancer.

Methods AK119 is a humanized antibody against CD73 and AK123 is a tetrameric bi-specific antibody targeting PD-1 and CD73. Binding assays of AK119 and AK123 to antigens, and antigen expressing cells were performed by using ELISA, Fortebio, and FACS assays. In-vitro assays to investigate the activity of AK119 and AK123 to inhibit CD73 enzymatic activity in modified CellTiter-Glo assay, to induce endocytosis of CD73, and to activate B cells were performed. Assay to evaluate AK123 activity on T cell activation were additionally performed. Moreover, the activities of AK119 and AK123 to mediate ADCC, CDC in CD73 expressing cells were also evaluated.

Results AK119 and AK123 could bind to its respective soluble or membrane antigens expressing on PBMCs, MDA-MB-231, and U87-MG cells with high affinity. Results from cell-based assays indicated that AK119 and AK123 effectively inhibited nucleotidase enzyme activity of CD73, mediated endocytosis of CD73, and induced B cell activation by upregulating CD69 and CD83 expression on B cells, and showed more robust CD73 blocking and B cell activation activities compared to leading clinical candidate targeting CD73. AK123 could also block PD-1/PD-L1 interaction and enhance T cell activation.

Conclusions In summary, AK119 and AK123 represent good preclinical biological properties, which support its further development as an anti-cancer immunotherapy or treating other diseases.

\section{REFERENCES}

1. Deaglio S, Dwyer KM, Gao W, Friedman D, Usheva A, Erat A, Chen JF, Enjyoji K, Linden J, Oukka M, et al. Adenosine generation catalyzed by CD39 and CD73 expressed on regulatory T cells mediates immune suppression. J Exp Med 2007; 204:1257-65.

2. Huang S, Apasov S, Koshiba M, Sitkovsky M. Role of A2a extracellular adenosine receptor-mediated signaling in adenosine-mediated inhibition of T-cell activation and expansion. Blood. 1997; 90:1600-10.

3. Novitskiy SV, Ryzhov S, Zaynagetdinov R, Goldstein AE, Huang Y, Tikhomirov OY, Blackburn MR, Biaggioni I,Carbone DP, Feoktistov I, et al. Adenosine receptors in regulation of dendritic cell differentiation and function. Blood 2008; 112:1822-31.

http://dx.doi.org/10.1136/jitc-2020-SITC2020.0702

\section{3 \\ ENGINEERED EXOSOMES WITH ALTERED CELLULAR TROPISM ACHIEVE TARGETED STING AGONIST DELIVERY AND SINGLE-AGENT TUMOR CONTROL IN VIVO}

Monique Kauke *, Nikki Ross, Dalia Burzyn, Shelly Martin, Ke Xu, Nuruddeen Lewis, Charan Leng, Su Chul Jang, Christine McCoy, Stephanie Yu, Kevin Dooley, Sriram Sathyanarayanan, Jonathan Finn. Codiak BiSciences, Cambridge, USA

Background Exosomes are natural, abundant extracellular vesicles capable of transferring complex molecules between neighboring and distant cell types. Translational research efforts have focused on co-opting this communication mechanism to deliver exogenous payloads to treat a variety of diseases. Important strategies to maximize the therapeutic potential of exosomes therefore include payload loading, functionalization of the exosome surface with pharmacologically active proteins, and delivery to target cells of interest.

Methods Through comparative proteomic analysis of purified exosomes, we identified several highly enriched and exosomespecific proteins, including a transmembrane glycoprotein (PTGFRN) belonging to the immunoglobulin superfamily. Leveraging PTGFRN as a scaffold for exosome surface display, we developed our engExTM platform to generate engineered exosomes functionalized with a variety of structurally and biologically diverse proteins.Systemically administered exosomes are primarily taken up by macrophages in the liver and spleen. To redirect exosome uptake to other cell types, we employed our engineering platform to display functional targeting ligands, including single domain antibodies, single chain variable fragments, single chain Fabs (scFabs), and receptor ligands, on the exosome surface at high density. To demonstrate that exosome surface modifications can alter cellular tropism, we generated exosomes displaying anti-Clec9A scFabs to target conventional type 1 dendritic cells (cDC1s), antiCD3 scFabs to target $\mathrm{T}$ cells, and CD40 ligand to target $\mathrm{B}$ cells. The engineered exosomes exhibited functional antigen binding that led to greater association with the cell types expressing the cognate receptor both in vitro and in vivo.

Results In mice, systemic administration of exosomes engineered to display scFabs targeting Clec9A resulted in a 4-fold increase in the percentage of cDC1 cells in the blood that had taken up exosomes over controls, and a 6-fold increase in the number of exosomes taken up per cell. We further showed that compared to untargeted exosomes, those with altered tropism achieved increased functional payload delivery to the target cell of interest. In primary mouse dendritic cells, anti-Clec9A exosomes loaded with a cyclic dinucleotide STING agonist achieved greater pathway induction, 2.3-fold greater as measured by IFN $\beta$ production, 2 -fold by IFN $\alpha$, and 15 -fold by IL-12, when compared to an untargeted control. Preliminary in vivo data show that intra-tumorally administered anti-Clec9A exosomes reduce the required STING agonist dose 10 -fold to achieve single-agent tumor control and induce immune responses against tumor-associated antigen, compared to controls.

Conclusions These results demonstrate the potential of our engExTM platform to generate targeted exosome therapeutics capable of immune cell stimulation and tumor growth inhibition in vivo. 